\title{
Oscillatory convection in binary mixtures: thermodiffusion, solutal buoyancy, and advection
}

\author{
D. Jung, P. Matura, and M. Lücke \\ Institut für Theoretische Physik, \\ Universität des Saarlandes, \\ D-66041 Saarbrücken, Germany
}

(Dated: February 5, 2020)

\begin{abstract}
The role of thermodiffusive generation of concentration fluctuations via the Soret effect, their contribution to the buoyancy forces that drive convection, the advective mixing effect of the latter, and the diffusive homogenisation are compared and elucidated for oscillatory convection. Numerically obtained solutions of the field equations in the form of spatially extended relaxed traveling waves, of standing waves, and of the transient growth of standing waves and their transition to traveling waves are discussed as well as spatially localized convective states of traveling waves that are surrounded by the quiescent fluid.
\end{abstract}

PACS numbers: 47.20.-k, 47.20.Ky, 47.54.+r, 47.27.Te 


\section{INTRODUCTION}

Convection in binary miscible fluids like ethanol-water, ${ }^{3} \mathrm{He}-{ }^{4} \mathrm{He}$, or various gas mixtures is an example of systems that show rich and interesting pattern formation behavior when driven away from equilibrium. It is paradigmatic for problems related to instabilities, bifurcations, selforganisation, complex spatiotemporal behavior, and turbulence. Compared to convection in one-component fluids the spatiotemporal properties are far more complex. The reason is that concentration variations which are generated via thermodiffusion, i.e., the Soret effect by externally imposed and by internal temperature gradients influence the buoyancy, i.e., the driving force for convective flow. The latter in turn mixes by advectively redistributing concentration. This nonlinear advection gets in developed convective flow typically much larger than the smoothening by linear diffusion - Péclet numbers measuring the strength of advective concentration transport relative to diffusion are easily of the order thousand. Thus, the concentration balance is strongly nonlinear giving rise to strong variations of the concentration field and to boundary layer behavior. In contrast to that, momentum and heat balances remain weakly nonlinear close to onset as in pure fluids implying only smooth and basically harmonic variations of velocity and temperature fields as of the critical modes. Without the thermodiffusive Soret coupling between temperature and concentration field initial concentration deviations from the mean diffuse away and influence no longer the balances of the other fields [such a system displays close to onset only the familiar stationary Benard rolls that are called for historical reasons states of stationary overturning convection (SOC)].

Hence, the feedback interplay between $(i)$ the Soret generated concentration variations, (ii) the resulting modified buoyancy, and (iii) the strongly nonlinear advective transport and mixing causes binary mixture convection to be rather complex with respect to its spatiotemporal properties and its bifurcation behavior.

Take for example the case of negative Soret coupling, $\psi<0$, between temperature and concentration fields [1]. Then the above described feedback interplay generates oscillations. In fact the buoyancy difference in regions with different concentrations was identified already in [4] as the cause for traveling wave convection.

Oscillatory convection appears in the form of the transient growth of convection at supercritical heating, in spatially extended relaxed nonlinear traveling wave (TW) and standing 
wave (SW) solutions that branch subcritically out of the conductive state via a common Hopf bifurcation, and in spatially localized traveling wave (LTW) states. TW and LTW convection has been studied experimentally and theoretically in detail [5, 6, 7, 8, 9, 10, 11, 12, 13, 14]. The transient oscillatory growth of convection was investigated by numerical simulations 15]. Nonlinear SW solutions were obtained only recently [16].

Here we compare these different oscillatory solutions and elucidate common features and differences.

In Sec. [I] we describe the system and our numerical methods for investigating it. Sec. III deals with extended states of TWs, transient growth of SWs, with SW $\rightarrow$ TW transitions, and with relaxed SWs. Then in Sec. IV LTWs are discussed. Sec. $\nabla$ contains a short conclusion.

\section{SYSTEM}

We consider a binary fluid layer of thickness $d$ at a mean temperature $\bar{T}$ and with a mean concentration $\bar{C}$ of the lighter component (e.g. ethanol in water). It is confined between two solid horizontal plates perpendicular to the gravitational acceleration $g$. The layer is heated from below with a temperature difference $\Delta T$ to the upper plate. The variation of the fluid density $\rho$ due to temperature and concentration variations is governed by the linear thermal and solutal expansion coefficients $\alpha=-\frac{1}{\rho} \frac{\partial \rho}{\partial T}$ and $\beta=-\frac{1}{\rho} \frac{\partial \rho}{\partial C}$, respectively. Both are positive for ethanol-water. The solutal diffusivity of the binary mixture is $D$, its thermal diffusivity is $\kappa$, and its viscosity is $\nu$.

The vertical thermal diffusion time $d^{2} / \kappa$ is used as the time scale of the system and all velocities are scaled by $\kappa / d$. Temperatures are reduced by the vertical temperature difference $\Delta T$ across the layer and concentration deviations from the mean concentration by $\frac{\alpha}{\beta} \Delta T$. The scale for the pressure is given by $\frac{\rho \kappa^{2}}{d^{2}}$. Then, the balance equations for mass, momentum, heat, and concentration [17, 18] read in Oberbeck-Boussinesq approximation [19]

$$
\begin{array}{r}
\boldsymbol{\nabla} \cdot \mathbf{u}=0 \\
\partial_{t} \mathbf{u}=-\boldsymbol{\nabla}(\mathbf{u}: \mathbf{u}+p-\sigma \boldsymbol{\nabla}: \mathbf{u})+\mathbf{B} ; \mathbf{B}=\sigma R(\delta T+\delta C) \mathbf{e}_{z} \\
\partial_{t} \delta T=-\boldsymbol{\nabla} \cdot \mathbf{Q}=-\boldsymbol{\nabla} \cdot[\mathbf{u} \delta T-\boldsymbol{\nabla} \delta T] \\
\partial_{t} \delta C=-\boldsymbol{\nabla} \cdot \mathbf{J}=-\boldsymbol{\nabla} \cdot[\mathbf{u} \delta C-L \boldsymbol{\nabla}(\delta C-\psi \delta T)] .
\end{array}
$$


Here, $\delta T$ and $\delta C$ denote deviations of the temperature and concentration fields, respectively, from their global mean values $\bar{T}$ and $\bar{C} ; \mathbf{Q}$ and $\mathbf{J}$ are the associated currents. $\mathbf{B}$ is the buoyancy. The Dufour effect [20, 21] that provides a coupling of concentration gradients into the heat current $\mathbf{Q}$ and a change of the thermal diffusivity is discarded in (2.3) since it is relevant only in few binary gas mixtures [22] and possibly in liquids near the liquid-vapor critical point [23].

Besides the Rayleigh number $R=\frac{\alpha g d^{3}}{\nu \kappa} \Delta T$ measuring the thermal driving of the fluid three additional numbers enter into the field equations: the Prandtl number $\sigma=\nu / \kappa$, the Lewis number $L=D / \kappa$, and the separation ratio $\psi=-\frac{\beta}{\alpha} \frac{k_{T}}{T}=-S_{T} \bar{C}(1-\bar{C}) \frac{\beta}{\alpha}$. Here $k_{T}=\bar{T} \bar{C}(1-\bar{C}) S_{T}$ is the thermodiffusion coefficient [17] and $S_{T}$ the Soret coefficient. They measure changes of concentration fluctuations due to temperature gradients in the fluid. $\psi$ characterizes the sign and the strength of the Soret effect. Negative Soret coupling $\psi$ (i.e., positive $S_{T}$ for mixture like ethanol water with positive $\alpha$ and $\beta$ ) induces concentration gradients of the lighter component that are antiparallel to temperature gradients. In this situation, the buoyancy induced by solutal changes in density is opposed to the thermal buoyancy. Throughout this paper we consider mixtures with $L=0.01, \sigma=10$, and various negative values of $\psi$ that are easily accessible with ethanol-water experiments.

When the total buoyancy exceeds a threshold, convection sets in - typically in the form of straight rolls. For sufficiently negative $\psi$ the primary instability is oscillatory [24]. Ignoring field variations along the roll axes we describe here $2 \mathrm{D}$ convection in an $x-z$ plane perpendicular to the roll axes with a velocity field

$$
\mathbf{u}(x, z, t)=u(x, z, t) \mathbf{e}_{x}+w(x, z, t) \mathbf{e}_{z} .
$$

This 2D type of convection is commonly enforced experimentally in convection channels of small extension in $y$-direction since the rolls are oriented preferentially perpendicular to the channel walls [2].

To find the time-dependent solutions of the partial differential equations describing convection we performed numerical simulations with a modification of the SOLA code that is based on the MAC method [25, 26]. This is a finite-difference method of second order in space formulated on staggered grids for the different fields. The Poisson equation for the pressure field that results from taking the divergence of (2.2) was solved iteratively using the artificial viscosity method [26]. In addition we used multi-mode Galerkin expansion 
methods [27].

The boundary conditions for the fields were as follows: we used realistic no slip conditions for the top and bottom plates at $z= \pm 1 / 2$ and we assumed perfect heat conducting plates. Furthermore, impermeability of the horizontal boundaries for the concentration was guaranteed by enforcing the vertical concentration current $\mathbf{e}_{z} \cdot \mathbf{J}$ to vanish at both plates

$$
\mathbf{e}_{z} \cdot \mathbf{J}=-L \partial_{z}(\delta C-\psi \delta T)(x, z= \pm 1 / 2 ; t)=0
$$

Laterally periodic extended convection structures were simulated by imposing lateral perodicity in $x$-direction with periodicity length $\lambda=2 \pi / k$. Spatially localized convection states were obtained in domains of size up to 160. To measure the strength of the thermal driving we use the reduced Rayleigh number $r=R / R_{c}^{0}$ that is scaled by the critical Rayleigh number $R_{c}^{0}$ for onset of pure-fluid convection with the critical wave number $k_{c}^{0}$. The analytical values are $R_{c}^{0}=1707.762$ and $k_{c}^{0}=3.11632$. However, to enable comparison with experimental, analytical, or numerical results we scale $R$ by the threshold $R_{c}^{0}$ in question, e.g., of the finite differences numerical code [28].

To characterize convection we monitored among others the evolution of the spatial maximum of the vertical velocity field $w_{\max }$, of the oscillation frequency, and of the reduced variance

$$
M=\sqrt{\left\langle\delta C^{2}\right\rangle /\left\langle\delta C_{\text {cond }}^{2}\right\rangle}
$$

of the concentration field. Note that $0 \leq M \leq 1$ measures to which extent the binary fluid is mixed. The better it is mixed the smaller is the spatial mean $\left\langle\delta C^{2}\right\rangle$ of the square of the concentration deviation from its mean - for spatially localized convection states $M$ is defined slightly differently, cf. Sec. IV] The boundary conditions, however, sustain always small Soret-induced concentration gradients against the action of advection and diffusion and prevents $M$ from vanishing completely. On the other hand, in the conductive state (denoted by the subscript "cond") with a Soret-induced vertical concentration stratification given by $\delta C_{\text {cond }}(z)=-\psi z$, there is no advective mixing. Its concentration variance, $\left\langle\delta C_{\text {cond }}^{2}\right\rangle=\psi^{2} / 12$, is largest yielding $M_{\text {cond }}=1$. 


\section{SPATIALLY EXTENDED OSCILLATORY CONVECTION}

The complexity of (oscillatory) convection in binary mixtures has been the subject of many studies [29] that are too numerous to be discussed here. A recent survey on papers adressing the role of the concentration field may be found in the introduction of [15].

In this section we compare characteristic properties of spatially extended oscillatory convection in the form of relaxed TWs 19, 30, 31], SWs [16] and of oscillatory transients 15] into, say, a nonlinear TW. In Fig. 1 we show the bifurcation diagrams of maximal vertical flow velocity $w_{\max }$ and oscillation frequency $\omega$ versus reduced Rayleigh number $r$ for the representative Soret coupling $\psi=-0.25$.

At $r_{\text {osc }}=1.335$ a SW solution as well as a pair of symmetry degenerate left and right traveling wave solutions bifurcate out of the conductive state with wave number $k=\pi$. At this Rayleigh number the system shows a subcritical Hopf bifurcation with a Hopf frequency $\omega_{H}\left(k=\pi, r_{\text {osc }}\right)=11.23$. Here two facts are worth mentioning: (i) the bifurcation threshold and the frequency are practically the same as for the critical waves with the critical wave number $k_{c}=3.135$ and (ii) TWs with wavelength $\lambda \simeq 2$ are often observed in large-scale experimental setups. This value corresponds to the minimum of $r_{o s c}(k)$. Fig. 2 shows $r_{\text {osc }}(k)$ for different $\psi$. The respective onset values for the phase velocities $v_{p}^{H}(k)=\omega_{H}(k) / k$ depend only weakly on the wave number $k$. The curves $v_{p}^{H}(k)$ are shifted to higher values with increasing negative $\psi$ due to the higher Soret-induced concentration contrast in the conductive state. The saddle node lines $r_{s}^{T W}(k)$ are more or less downwards shifted versions of $r_{\text {osc }}(k)$. The size of the interval, $r_{\text {osc }}(k)-r_{s}^{T W}(k)$, of subcritical TWs increases when $\psi$ becomes more negative. For the parameters of Fig. 2 the TW branches end at large amplitudes in a well mixed state of vanishing frequency by merging with the stationary SOC solution branch of the same wavelength. For the parameters of Fig. 1 this happens at $r^{*}=1.65$ where the SOC solution branch is included for convenience as well.

In contrast to the TW solutions, the SWs remain unstable at least against a transition into a (transient) TW. Their solution branches (cf. Sec. IIID) end in period dubling cascades [16]. 


\section{A. Advective mixing and diffusion of concentration fluctuations}

When moving along the TW and SW solution branches in Fig. 11 the frequency $\omega$ and the TW phase velocity $v_{p}=\omega / k$ decrease monotonically starting from the large Hopf value at the bifurcation threshold. This is related to the advective reduction of the Soret generated concentration gradients. In fact there is a universal linear relation between the degree of mixing and the frequency $\omega$ which holds for weakly nonlinear as well as for strongly nonlinear states and for transients alike. See for example [30, 39] for TWs, 14] for LTWs, and [15] for transients. This scaling relation $\omega / \omega_{H} \simeq M$ holds also for SWs as one can infer from Fig. 3. Also TWs with wavelength different from 2 show roughly this scaling.

In particular on the stable, strongly nonlinear TW solution branch the binary fluid gets with increasing $w_{\max }$ and thermal driving $r$ more and more mixed and $M$ reduces almost to zero - only concentration gradients in the narrow boundary layers survive. This holds even more so for the well mixed SOC states (see e.g. the SOC2 in Fig. 4 which is marked in Fig. 1 by the filled triangle) that resemble closely the corresponding stationary state in the pure fluid with the same $\sigma$ and $r$.

The transition to convection at $r_{o s c}$ is hysteretic, i.e., of first order because the Soret coupling coefficient $\psi=-0.25$ is sufficiently negative. The associated precipitous growth of convection is caused by an interplay between the solutal contribution to the buoyancy [19, 30] that tends to stabilize the conductive state and the effect of advective mixing. The latter enhances convection by reducing the adverse effect of the Soret generated concentration variations.

There occurs an "S"-shaped deformation of the unstable part of the TW bifurcation branch [dashed (blue) line in Fig. 1] when the advection velocity $w_{\max }$ has grown to become equal to the TW phase velocity $v_{p}$. Then the first closed streamlines appear [31] in the frame of reference that is comoving with the TW phase velocity and where the TW solution is time independent. This is indeed a characteristic feature of strongly nonlinear TW convection, cf. further below.

For $w_{\max }<\left|v_{p}\right|$, i.e., closer to the threshold $r_{o s c}$ all streamlines are open and the field structures look almost harmonic - see the TW1 plot in Fig. 4. This weakly nonlinear TW, marked by an open circle in Fig. 1, is just at the border $w_{\max } \simeq\left|v_{p}\right|$. This condition marks also the border line beyond which a straightforward small-amplitude expansion around the 
convective onset breaks down [39]. In addition the generic transient growth dynamics of oscillatory convection (consisting initially of oppositely traveling waves of roughly equal velocity amplitudes $\left.\left|A_{R}\right| \simeq\left|A_{L}\right|\right)$ undergoes a dramatic change that cannot be described at all with amplitude equation models when the flow amplitudes $\left|A_{R, L}\right|$ approach the border line value of $\omega / k[15]$.

The qualitative change in the flow topology between weakly and strongly nonlinear TWs causes a different mixing behavior with increasing amplitude. This is the reason for the "S"shaped deformation of the unstable part of the TW bifurcation branch [dashed (blue) line in Fig. 1]. It becomes more pronounced with $\psi$ becoming more negative (see e.g. Fig. 9). Eventually the strongly nonlinear TW solution branch develops a bistable part there on which fast TWs are located [30, 39].

For relaxed TWs with $0<\left|v_{p}\right|<w_{\max }$ the remaining open streamlines are spatially correlated with inner boundary layers of the concentration field. As indicated in the TW2 of Fig. 4 they meander along the (green) regions of mean concentration, $\delta C=0$, between and around the roll-like regions of closed streamlines. In a right-propagating TW the regions of closed streamlines for the right (left) turning fluid domains are rich (poor) in the lighter component - here ethanol - and they are displaced towards the upper cold (lower warm) plate, where the Soret effect maintains a boundary layer with alcohol surplus (deficiency). In addition the meandering open streamlines lie between the upper (lower) closed streamline regions and the opposite bottom (top) concentration boundary layer. This structure of closed and open streamlines causes the top (bottom) boundary layer to feed high (low) concentration only into the right (left) turning roll domain at the location of downflow (upflow). Then the fluid becomes diffusively homogenized in the closed streamline regions of the rolls leading to anharmonic concentration profiles of trapezoidal shape shown in the top plot of the left column of Fig. 4. The motion of the rolls with their specific concentration distribution implies a mean concentration current which is directed to the right in the upper and to the left in the lower half of the layer. See refs. [19, 40, 41] for a more detailed discussion of this current.

With increasing $w_{\max }$ and decreasing $\left|v_{p}\right|$ the regions of closed streamlines grow at the expense of the open ones. Thereby, the former also come closer to the respective opposing boundary layer. This decreases the asymmetry of the boundary layer feeding into oppositely turning rolls. As a consequence the concentration contrast between adjacent TW rolls 
decreases until in the SOC state with $v_{p}=0$ the rolls are fed symmetrically by both boundary layers and mirror symmetry between the rolls is established.

\section{B. Symmetries}

SOCs and SWs are laterally mirror symmetric around positions of maximal up- and downflow, e.g. $x=0$ in Fig. 4 and the node locations of the fields are fixed in time. This symmetry is broken in TWs - see, e.g., the concentration contrast between left and right turning rolls in Fig. 4. But all fields of SOCs, SWs, and TWs have at every instant definite parity under the mirror-glide $(\mathrm{MG})$ operation $(x, z) \rightarrow(x+\lambda / 2,-z)$ of vertical reflection at mid-height, $z=0$, combined with lateral translation by half a wavelength. We did not observe SWs without this symmetry - perturbations breaking it that we introduced for test purposes always decayed rapidly to zero.

Furthermore, all transients investigated in [15] obeyed the MG symmetry with the exception of the very early stage in cases where the imposed initial conditions were not MG symmetric. But even then the MG symmetry was rapidly restored by a fast decay of MG symmetry-breaking modes. Also the transient growth seen in the experiments in an annular geometry by Winkler and Kolodner [42] was locally MG symmetric. A time dependent generalization of this symmetry was found to be realized in LTW states [43] and an extension to 3D patterns was observed in [4]. Furthermore, MG symmetric convective structures were not only observed in Soret driven convection with only temperature gradients imposed but also in thermosolutal convection [45].

Thus, the MG symmetry that is displayed by the basic conductive state and by the linear critical convective modes seems to be quite robust and also persistent in non-linear convective structures of pure fluids and of mixtures. The robustness of this symmetry is remarkable given that the nonlinearity in the concentration balance, i.e., the Peclet number $w / L$ is quite large - of the order of 1000. However, the concentration field is "tamed" by being coupled to the velocity and temperature fields. Their shape remains mostly harmonic for small supercritical thermal driving like in pure fluid convection. And thus the increase in structural complexity associated with a MG symmetry breaking does not occur.

Finally, the SW fields start at onset to have in addition a definite mirror-timeshift symmetry (MTS), e.g., $f(x, z, t)=-f(x,-z, t+\tau / 2)$ for $f=\delta C, \delta T$, and the vertical veloc- 
ity field $w$ with $\tau=2 \pi / \omega$ being the SW oscillation period. At mid-height the condition $f(t)=-f(t+\tau / 2)$ implies in particular that positive and negative field extrema of an oscillation cycle have equal magnitudes. SWs with smaller frequency that are located in the end region of the SW solution branches break this symmetry (cf. Sec. IIID). This is a prerequisite for period doubling [46].

\section{Supercritical growth and SW $\rightarrow$ TW transition}

Here we review the supercritical spatiotemporal behavior of SW transients. We start from the quiescent conductive state at $r<r_{\text {osc }}$, disturb it slightly by adding small random numbers in the range of $\left[-10^{-4}, 10^{-4}\right]$, and simultaneously increase the control parameter $r$ slightly above threshold as indicated in Fig. 1. Then one observes a generic transition scenario that occurs similarly also in analogous experiments. It consists of three evolution phases that we have found to be generic for convective growth out of small unspecific perturbations: $(i)$ an exponentially growing SW of high frequency over a time interval the length of which depends on the size of the initial perturbations of the conductive state, ( $i i)$ an intermediate phase that is always very short with a spatiotemporally complicated transformation from SW into a high-frequency TW and finally (iii) a long-term TW transient to a low-frequency, strongly nonlinear, relaxed TW or SOC state depending on $r$ [15].

In generic, i.e., non specific initial perturbations that break the mirror symmetry $x \rightarrow-x$ only weakly the two critical oscillatory modes of right and left traveling waves that can grow above the Hopf threshold are contained with roughly equal amplitudes, i.e., $\left|A_{R}\right| \simeq\left|A_{L}\right|$. Thus, initially, i.e., as long as linear theory applies, the two critical TW modes that started with $\left|A_{R}\right| \simeq\left|A_{L}\right|$ grow exponentially and independently of each other with the same growth rate; and their superposition causes SW-like oscillations with the large critical frequency as in Fig. [5] a. Hence, "almost" mirror symmetric experimental setups that do not favor a particular TW propagation direction cause an initial growth phase with SW characteristics 42].

But then a competition between the two TW constituents sets in when the advective nonlinearities have become sufficiently strong. They amplify the mirror-symmetry breaking differences between $\left|A_{R}\right|$ and $\left|A_{L}\right|$ and cause the decay of the minority TW. Thus, the SW, which still has a large frequency, is transformed into a fast TW (Fig. 5 b $-\mathrm{h}$ ). This SW $\rightarrow$ TW 
transformation being advection driven is spatiotemporally complicated, in particular for the concentration field and it implies a dramatic redistribution of concentration by advective "rolling in" of concentration (Fig. 5 c -g). It takes place within less than one vertical thermal diffusion time and it starts roughly when the flow amplitudes of the two constituent TWs have grown to about the phase velocity $v_{p}$ : First, concentration is advected up- and downwards in the form of plumes by the growing SW-like flow that reverts periodically its direction; the vertical concentration gradient being still as large as that of the quiescent conductive state. The $\mathrm{SW} \rightarrow \mathrm{TW}$ transformation is triggered by an advective wave breaking and wave toppling process of the crests and troughs of the concentration wave (Fig. [5]c-d), whereas the waves of $w$ and $\delta T$ do not undergo substantial structural changes. Advective nonlinearities have by now become sufficiently strong to make the mirror-symmetry-breaking differences between the original, left and right propagating TW constituents of the SW clearly visible. When the concentration wave crests (troughs) with a high (low) alcohol content bend and topple, they are advectively "rolled in".

The flow induced sequence of first deforming, then bending, and finally rolling in the plume-like wave crests and troughs is associated with and driven by a growth of the spatial phase shift between velocity and concentration field from zero to about $\lambda / 4$ during the SW $\rightarrow$ TW transformation: In the SW the spatial location of the nodes of $\delta C$ and $w$ coincide while their oscillations are shifted in time by about a quarter of an oscillation period. The value of this phase difference, $\varphi_{C}-\varphi_{w} \simeq \pi / 2$, does not change during the whole transition sequence but in the TW, it also implies a spatial shift of $\delta C$ and $w$ of $\lambda / 4$.

In the early TW phase (Fig. 5 h) the lateral concentration difference between adjacent roll like regions is still almost as large as the initial vertical concentration contrast in the conductive state. Thus, the frequency and the phase velocity of this emerging TW are still very large, i.e., not much smaller than the critical values. But then for the parameters of Fig. 5 a long-term TW transient to a low-frequency, strongly nonlinear and strongly anharmonic relaxed TW state (Fig. [5i) sets in: slow diffusion degrades and homogenizes the concentration striations, the spatial extension over which $\delta C$ is constant at the two alternating high and low levels increases, the plateau height decreases, the width of boundary layers between these plateaus shrinks, the $\delta C$-wave profile becomes more and more trapezoidal, and the alcohol surplus (deficiency) in the cold top (warm bottom) part of the fluid layer decreases, thereby reducing the overall vertical concentration difference between top and bottom. This 
longtime degradation of concentration gradients is reflected by a dramatic decrease of the mixing number $M$ and with it of the frequency $\omega$ relative to the initial values - the better the fluid becomes mixed the smaller is $\omega$.

\section{Standing waves}

TW and LTW convection has been studied experimentally and theoretically in detail 5, 6, 7, 8, 9, 10, 11, 12, 13, 14]. But little is known about nonlinear SW states beyond a weakly nonlinear analysis [47] that is restricted to the immediate vicinity of the oscillatory threshold. It showed that SWs are unstable there, typically bifurcating backwards.

In order to obtain the solution branch, we stabilize the SW states by suppressing phase propagation (so TWs can not compete against SWs) and, if necessary, by exerting a control procedure. The latter operates via the field amplitudes or the heat current injected into the fluid and the Rayleigh number in response to the instantaneous frequency $\omega$ and its temporal derivative $\Delta \omega / \Delta t$, respectively [48]. In this way we trace out the SW solution branch all the way from close to onset with large frequency to slowly oscillating SWs that eventually period-double into chaos.

Fig. 6] shows the bifurcation behavior of SWs and how it changes with varying Soret coupling strength. The solution branch for the SOC is included for comparison only for $\psi=$ -0.03. The heating range in which SWs exist increases when $\psi$ becomes more negative since the oscillatory bifurcation threshold $r_{\text {osc }}$ is shifted stronger to higher $r$ than the SW saddlenode positions at $r_{s}^{S W}$ which marks the lower end of the $r$-interval containing SWs. All these SWs bifurcate subcritically out of the conductive state as unstable solutions. They become stable via saddle-node bifurcations when the phase-pinning condition is imposed. However, when this condition is lifted completely then SWs decay by developing TW transients since any spatial phase difference between $\delta C$ and $w$ causes the extrema of the latter to be "pulled" towards the solutally shifted buoyancy extrema. Depending on $r$ these transients either end in a nonlinear TW or SOC or the conductive state.

Moving along an SW branch the maximal vertical upflow velocity $w_{\max }$ [Fig. 6(a)] does not increase monotonically as in TWs and SOCs but rather has a maximum somewhat below the respective SOC value before it drops again. On the other hand, $\omega$ and $M$ decrease monotonically starting with the Hopf frequency $\omega_{H}$ [upper end of the curves in Fig. [6(b)] and 
$M=1$, respectively, at onset. This variation follows the universal scaling law $\omega / \omega_{H} \simeq M$, cf. Fig. 3 ,

The typical bifurcation behaviour of two representative SWs of Fig. [6 with $\psi=-0.03$ and -0.25 is displayed in more detail in Fig. [7. Full (dashed) lines refer to stable (unstable) branches. Note that the large-amplitude upper SOC solution branches [the one for $\psi=-0.03$ is shown explicitly in Fig. [7(a)] are stable down to their respective saddle-nodes when phase propagation is suppressed so that TW solutions do not exist. Then, the SW solutions, too, change their stability at saddle-node bifurcation(s), one for $\psi=-0.03$ at $r_{s}^{S W} \approx 1.0373$ and three for $\psi=-0.25$ [two of them can be seen in Fig. [7(b) at $r_{s}^{S W} \approx 1.1238$ ]. With increasing $r$ the flow amplitude of the stable SW slightly decreases. Then the MTS breaks. Thereafter, for example, the downflow (upflow) extrema occurring in the SW oscillations, say, at $x=0$ $( \pm \lambda / 2)$ are more intense than the upflow (downflow) extrema. This is reflected by the first splitting of the SW solution branches in Fig. [7. Consequently, the time averaged fields have now a net SOC-like structure with non zero mean downflow (upflow), say, at $x=0( \pm \lambda / 2)$.

In Fig. 8 we show for $\psi=-0.03$ how MTS breaking changes the SW phase dynamics using $w, \dot{w}$ at the mid position $x=0=z$ and $M$ as characteristic local and global quantities, respectively. By definition $M$ oscillates with twice the SW frequency as long as MTS holds (dash-dotted lines in Fig. [8). The particular MTS-broken SW orbits of Fig. 8] move closer to the low-amplitude fixed point on the lower SOC solution branch of Fig. I(a) with downflow at $x=0$, so the SW spends more time in the downflow phase at $x=0$ than in the upflow phase. This can be seen more clearly in the temporal oscillation profile of $w$ (Fig. 4 in [16]) ; it develops a plateau close to the value of the corresponding SOC fixed point.

Then, at a certain $r$ depending on $\psi$, the first period-doubling occurs followed by further subsequent period-doublings that lead to chaos (Fig. [7] and Fig. 8). For stronger Soret coupling, i.e. $\psi=-0.25$ [Fig. [7(b)], we could also resolve a $r$-window with period-3 SW states and subsequent period doublings.

Beyond the last chaotic windows, we did not observe any stable SWs: heating above this threshold leads to the development of transients into a stable, large-amplitude SOC on the upper solution branch [e.g., with amplitude $w_{S O C} \simeq 2.51$ in Fig. 7)(a), i.e., well outside the plot range of Fig. 8 .

Thus, here the question arises whether - and if so - how and how long the heteroclinic orbits connecting the two unstable symmetry degenerate SOC fixed points on the lower 
solution branch with small convection amplitudes $\left(w_{S O C} \simeq 0.71\right.$ in Fig. 8) organize and restrain the dynamics of the periodic and chaotic SWs that switch between up- and downflow.

\section{LOCALIZED TRAVELING WAVES}

LTWs consist of wave trains of traveling convection rolls which are surrounded by quiescent fluid [14, 43, 49, 50]. There exist several attemps to model LTWs by weakly nonlinear small-amplitude expansions around the convective onset [51, 52] and modifications thereof 53, 54, 55]. But due to the strongly nonlinear characteristics of LTWs these models are aimed at some qualitative aspects of LTW states.

Here we investigate stable LTWs at subcritical driving, $r<r_{\text {osc }}$, where the quiescent conductive surrounding is stable as well.

The rolls grow out of this environment at the tail end of the wave train, travel through the convective bulk of the LTW with increasing phase velocity $v_{p}(x)$ and wavelength $\lambda(x)$, and decay at the head of the wave train (cf. Fig. 10). These stable LTW states are uniquely selected. Their width $l$ (or number of rolls) is stationary and depends in an unique way on the control parameters (cf. Fig. 9). The whole convective region drifts through the motionless state with a drift velocity $v_{d}$ that can be positive or negative but that is small compared to the phase velocity 14]. Therefore, the frequency of a LTW is constant in a frame of reference that is comoving with the LTW's drift.

To characterize the concentration variations in a LTW we use a local mixing number $M(x)$ that is defined similarly to the case of extended states as the mean variance of $\delta C$. But here the lateral average is replaced by a time average at a fixed lateral position $x$,

$$
M(x)=\sqrt{\left\langle\delta C^{2}\right\rangle /\left\langle\delta C_{\text {cond }}^{2}\right\rangle} .
$$

So brackets denote here an average over $z$ and $t$ instead of $z$ and $x$ as in Eq. (2.7). Fig. 10 (c) shows the variation of $M(x)$ over the convective region of a typical long LTW. The relation between $M(x)$ and the local phase velocity $v_{p}(x)$ in the bulk of the LTW is the same as the relation between $M$ and $v_{p}=\omega / k$ for TWs.

The smallest stable localized pulses have for each $\psi$ a minimal width of about 5 rolls. With increasing $r$ LTWs grow in width and amplitude and $l$ diverges at a maximal driving $r_{\max }$. Beyond this value, LTWs of stationary width seem to be no longer possible. Thereafter, only 
transient localized convective regions can occur which keep expanding between two fronts.

As one can see in Fig. 9 the existence interval of LTWs at fixed $\psi$ can be divided into two regimes: In the regime of short pulses the frequency decreases with increasing $r$ and the amplitude grows fast whereas in the regime of long LTWs the frequency increases with $r$ and the amplitude grows only moderately.

Short LTWs are dominated by a direct interaction between the growth part and the decay part of the convection rolls. Long LTWs are characterized by an extended bulk part where rolls travel with phase velocity $v_{p}(x)$ that increases from tail to head. This corresponds to an increasing local wavelength $\lambda(x)=2 \pi v_{p}(x) / \omega$ since the frequency $\omega$ is constant in the frame that is comoving with $v_{d}$. The speeding up of $v_{p}(x)$ reflects the growing concentration contrast between adjacent rolls along the $x$-direction: The minimal mixing number is located in the growth region of the tail where $v_{p}(x)$ and $\lambda(x)$ are minimal as well. On their way from tail to head the rolls do not reach a stationary balance between $\delta C$ injection from the different boundary layers and advective mixing and diffusive homogenisation of concentration differences on a constant level of small $M$. Rather LTW rolls collapse at the head when $v_{p}$ has grown up to $w_{\max }$ [right arrow in Fig. 10 (b)]. Thereafter concentration is discharged and sustains a barrier of $\langle\delta C\rangle$ ahead of the decay part which stabilizes the conductive state there against invasion of convection.

Fig. 9 (b) and (c) compare the maximal flow velocities and the frequencies of LTWs for several $\psi$ with the respective TW branch of $\lambda \simeq 2$ for which the saddle location $r_{s}^{T W}$ is the lowest one. Note that the LTW values for $w_{\max }$ and $\omega$ always lie near the saddle values of TWs. Although there exist no extended TWs below the shown TW saddle positions there exist stable LTWs ahead of these global TW saddles when $\psi$ is sufficiently negative. For example, for $\psi=-0.4$ almost all LTWs of finite width appear below the existence region for TWs as can be observed in Fig. 9 .

The intriguing existence of stable LTWs without coexisting TWs is ensured by a flowinduced lateral concentration redistribution over its convective bulk. Positive "blue" (negative "red") concentration deviation from the global mean is sucked from the top (bottom) boundary layer into right (left) turning rolls as soon as they become nonlinear during their growth [left arrow in Fig. 10 (b)]. Convection in the bulk of a (long) LTW shows all the characteristics of a strongly nonlinear extended TW. Thus, as discussed in Sec. IIIA positive (negative) concentration is transported within the closed streamline regions predominantly 
in the upper (lower) half away from the tail towards the head. This lateral concentration transport is reflected in the time averaged current of $\delta C$ [cf. its streamlines in Fig. 10(e)]. At the same time, mean concentration, $\delta C \simeq 0$, migrates mostly to the left along open velocity field streamlines that meander between the closed roll regions [green regions in Fig. 10(a)]. In this way a region with well mixed fluid, i.e., almost vanishing $\delta C$ is created and sustained under the tail. This strongly nonlinear large scale concentration redistribution that maintains the concentration locally at a homogeneous mean level under the trailing front makes there the growth of convection possible: By reducing the stabilizing concentration gradients the driving buoyancy force is locally increased there to levels which suffice to cause convection in a well-mixed fluid.

Fig. 10 (d) shows that this effect causes the mean convectively generated $C$ profile to extend significantly further into the conductive region than the mean convective temperature field. Thus, the buoyancy $\langle b\rangle$ [cf. Fig. 10] (d)] is determined in the front regions predominantly by the concentration field. This explains (i) the decrease of buoyancy below conduction levels ahead of the decay part with the associated restabilization of conduction there and (ii) the increase of $\langle b\rangle$ out of the conductive state at the tail and its strong overshoot over the bulk enabling convection growth even for heating $r$ where no stable extended TWs are possible.

\section{CONCLUSION}

Three oscillatory steady states of convection in binary fluid mixtures like ethanol-water were investigated and compared numerically in a cross section perpendicular to the roll axes: Traveling waves, standing waves, and localized traveling waves. In addition the supercritical growth of SWs out of perturbations and the $\mathrm{SW} \rightarrow \mathrm{TW}$ transformation was elucidated.

The full bifurcation behavior of TWs and SWs has been presented and their typical concentration field structures are shown in detail. Both extended oscillatory periodic patterns bifurcate subcritically out of the conductive state in a common Hopf bifurcation. The decrease of the phase velocity or the frequency along the solution branches is connected to the decrease of the mean $\delta C$ contrasts in the system due to advective mixing. Thereby, the different symmetries and flow topologies of TWs and SWs cause a different behavior in the strongly nonlinear regime. However frequencies and mixing numbers are always related to 
each other via the universal scale relation $\omega / \omega_{H} \simeq M$.

TWs are mirror-glide symmetric under $(x, z) \rightarrow(x+\lambda / 2,-z)$. At high amplitudes they are characterized by the occurrence of closed streamlines in the frame comoving with the TW's phase velocity. The regions of closed streamlines are displaced alternately towards the upper and lower plates with their respective concentration boundary layers. The latter feed selectively concentration only into the nearby closed streamline domains where it is homogenized. This leads to the characteristic concentration distribution with homogeneous rolls of alternating signs in $\delta C$. They are separated by meandering inner boundary layers of $\delta C \sim 0$ which are spatially correlated with open streamlines of the velocity field. With increasing amplitude the concentration contrasts between the rolls decrease, the phase velocity slows down, and the TW ends at large amplitudes in a mirror symmetric SOC state.

SWs are always laterally mirror symmetric. The solution branch turns around at a saddle node and becomes stable there under phase pinning conditions. For strongly negative $\psi$ the stable part of the SW branch is directed towards lower amplitudes - in contrast to the TW case - and develops further saddle nodes. The complicated bifurcation behavior at low frequencies seems to be due to an interaction with the unstable low amplitude SOC solutions. There is a breaking of the mirror-timeshift symmetry which is a prerequisite for the following period doubling cascade into chaos. Without phase pinning the SWs develop TW transients which end either in a nonlinear TW or a SOC state depending on the parameters. This mechanism is similar to the symmetry breaking of a weakly nonlinear SW during its transient growth at supercritical heating towards a nonlinear TW. The transition is characterized by a dramatic advective reorganization of the $\delta C$ field and therefore of the buoyancy field within a short time interval.

The only global symmetry operation for a LTW is the mirror-timeshift $[(x, z, t) \rightarrow$ $(x,-z, t+\tau / 2)]$ in the frame which is comoving with its small drift velocity $v_{d}$. The structual properties of a generic long LTW were presented as well as the global bifurcation behavior with $r$ for different $\psi$. For strongly negative separation ratios LTWs exist monostably at low heatings $r$ where no extended TWs are possible. This is ensured by a flow-induced lateral large scale redistribution of concentration over its TW dominated convective bulk: Thereby, concentration is maintained under the tail of the wave train at a homogeneous mean level. This increases the driving buoyancy forces there locally to levels which suffice 
to cause convection.

[1] For 5 weight percent of ethanol mixed into water at $T=20^{\circ} \mathrm{C}$ the separation ratio measuring the Soret coupling strength [2] is $\psi \simeq-0.3[3]$.

[2] M. C. Cross and P. C. Hohenberg, Rev. Mod. Phys. 65, 851 (1993) and references cited therein.

[3] P. Kolodner, H. L. Williams, and C. Moe, J. Chem. Phys. 88, 6512 (1988).

[4] R. W. Walden, P. Kolodner, A. Passner, and C. M. Surko, Phys. Rev. Lett. 55, 496 (1985).

[5] See ref. 2] and references, e.g., in [15, 30].

[6] R. Heinrichs, G. Ahlers, and D. S. Cannell, Phys. Rev. A 35, 2761 (1987); K. Lerman, D. S. Cannell, and G. Ahlers, Phys. Rev. E 59, 2975 (1999).

[7] K. E. Anderson and R. P. Behringer, Physica D 51, 444 (1991).

[8] B. L. Winkler and P. Kolodner, J. Fluid Mech. 240, 31 (1992); P. Kolodner, Phys. Rev. E 50, 2731 (1994).

[9] H. Touiri, J. K. Platten, and G. Chavepeyer, Eur. J. Mech. B 15, 241 (1996).

[10] E. Moses, J. Fineberg, and V. Steinberg, Phys. Rev. A35, 2757 (1987); E. Kaplan, E. Kuznetsov, and V. Steinberg, Phys. Rev. E 50, 3712 (1994).

[11] C. M. Surko, D. R. Ohlsen, S. Y. Yamamoto, and P. Kolodner, Phys. Rev. A 43, 7101 (1991); C. M. Aegerter and C. M. Surko Phys. Rev. E 63, 46301 (2001).

[12] L. Z. Ning, Y. Harada, and H. Yahata, Prog. Theor. Phys. 98, 551 (1997).

[13] O. Batiste, E. Knobloch, I. Mercader, and M. Net, Phys. Rev. E. 65, 016303 (2001).

[14] D. Jung and M. Lücke, Phys. Rev. Lett. 89, 054502 (2002).

[15] C. Fütterer and M. Lücke, Phys. Rev. E 65, 036315 (2002).

[16] P. Matura, D. Jung, and M. Lücke, Phys. Rev. Lett. 92, 254501 (2004).

[17] L. D. Landau and E. M. Lifschitz, Hydrodynamik, (Akademie-Verlag, Berlin, 1966).

[18] J. K. Platten and J. C. Legros, Convection in Liquids, (Springer, Berlin, 1984).

[19] W. Barten, M. Lücke, M. Kamps, and R. Schmitz, Phys. Rev. E 51, 5636 (1995).

[20] W. Hort, S. J. Linz, and M. Lücke, Phys. Rev. A 45, 3737 (1992).

[21] St. Hollinger and M. Lücke, Phys. Rev. E 52, 642 (1995).

[22] J. L. Liu and G. Ahlers, Phys. Rev. Lett. 77, 3126 (1996).

[23] G. W. T. Lee, P. Lucas, and A. Tyler, J. Fluid Mech. 135, 235 (1983). 
[24] The oscillatory instability is not primarily due to concentration diffusion being small: even if the diffusive time scales for momentum, heat, and concentration are the same $(L=\sigma=1)$ there are oscillations. They are caused by a sufficiently strong "off diagonal" Soret coupling between the degrees of freedom of concentration and temperature and their feedback into the buoyancy.

[25] J. E. Welch, F. H. Harlow, J. P. Shannon, and B. J. Daly, Los Alamos Scientific Laboratory of the University of California, Report No. LA-3425, 1966 (unpublished); C. W. Hirt, B. D. Nichols, and N. C. Romero, Los Alamos Scientific Laboratory of the University of California, Report No. LA-5852, 1975 (unpublished).

[26] R. Peyret and T. D. Taylor, Computational Methods in Fluid Flow (Springer, Berlin, 1983).

[27] B. Huke and M. Lücke, in Thermal nonequilibrium phenomena in fluid mixtures, edited by W. Köhler and S. Wiegand, Lecture Notes in Physics, 584 (Springer, Berlin, 2002), p. 334.

[28] For a discretization of $\mathrm{dx}=1 / 20(\mathrm{dx}=1 / 40)$ the threshold for a one-component fluid is $R_{c}^{0}=$ $1686\left(R_{c}^{0}=1702\right)$; Ch. Jung, Ph. D. thesis, Universität des Saarlandes, Saarbrücken, 1997.

[29] For a review see 2, 18]. Further references may be found, e.g., in [19, 30]. See also the more recent publications [32, 33, 34, 35, 36, 37, 38] of some of the many groups that have investigated convection in binary mixtures.

[30] M. Lücke, W. Barten, P. Büchel, C. Fütterer, St. Hollinger, and Ch. Jung, in Evolution of Structures in Dissipative Continuous Systems, edited by F. H. Busse and S. C. Müller, Lecture Notes in Physics, m55 (Springer, Berlin,1998), p. 127.

[31] St. Hollinger and M. Lücke, Phys. Rev. E 57, 4238 (1998).

[32] E. Kaplan, E. Kuznetsov, and V. Steinberg, Phys. Rev. E 50, 3712 (1994).

[33] P. Kolodner, J. A. Glazier, and H. Williams, Phys. Rev. Lett. 65, 1579 (1990); P. Kolodner, G. Flätgen, and I. G. Kevrekidis, ibid 83, 730 (1999).

[34] H. Touiri, J. K. Platten, and G. Chavepeyer, Eur. J. Mech. B 15, 241 (1996).

[35] L. Z. Ning, Y. Harada, and H. Yahata, Prog. Theor. Phys. 98, 551 (1997).

[36] K. Lerman, E. Bodenschatz, D. S. Cannell, and G. Ahlers, Phys. Rev. Lett. 70, 3572 (1993); K. Lerman, G. Ahlers, and D. S. Cannell, Phys. Rev. E 53, R2041 (1996); K. Lerman, D. S. Cannell, and G. Ahlers, ibid 59, 2975 (1999).

[37] C. M. Aegerter and C. M. Surko Phys. Rev. E 63, 46301 (2001).

[38] O. Batiste, M. Net, I. Mercader, and E. Knobloch, Phys. Rev. Lett. 86, 2309 (2001). 
[39] St. Hollinger, P. Büchel, and M. Lücke, Phys. Rev. Lett. 78, 235 (1997).

[40] W. Barten, M. Lücke, W. Hort, and M. Kamps, Phys. Rev. Lett. 63, 373 (1989).

[41] W. Barten, M. Lücke, and M. Kamps, Physica D 61, 183 (1992).

[42] B. L. Winkler and P. Kolodner, J. Fluid Mech. 240, 31 (1992).

[43] W. Barten, M. Lücke, and M. Kamps, Phys. Rev. Lett 66, 2621 (1991); W. Barten, M. Lücke, M. Kamps, and R. Schmitz, Phys. Rev. E 51, 5662 (1995).

[44] Ch. Jung, B. Huke, and M. Lücke, Phys. Rev. Lett. 81, 3651 (1998).

[45] A. Spina, J. Toomre, and E. Knobloch, Phys. Rev. E 57, 524 (1998).

[46] J. W. Swift and K. Wiesenfeld, Phys. Rev. Lett. 52, 705 (1984).

[47] W. Schöpf and W. Zimmermann, Phys. Rev. E 47, 1739 (1993).

[48] We drive the system towards the unstable limit cycle (ULC) with frequency $\omega_{*}$ that is located on the subcritical SW solution branch as follows: To reduce deviations with growing (decreasing) convection amplitude $A$ that decrease (increase) $\omega$ at fixed $r$ we set $A_{n+1}=$ $A_{n}\left[1+W_{A}\left(\omega-\omega_{*}\right)\right]$. Furthermore, when $\Delta \omega / \Delta t$ is negative (positive) we move towards the SW solution branch by decreasing (increasing) $r$ according to $r_{n+1}=r_{n}\left(1+W_{r} \Delta \omega / \Delta t\right)$. The control depends on the actual values of $\omega-\omega_{*}$ and $\Delta \omega / \Delta t$ and vanishes only at the ULC with the preselected frequency $\omega_{*}$. The optimal weight factors $W_{A}$ and $W_{r}$ depend on $\omega_{*}$.

[49] E. Moses, J. Fineberg, and V. Steinberg, Phys. Rev. A35, 2757 (1987); R. Heinrichs, G. Ahlers, and D. S. Cannell, ibid. 2761 (1987); P. Kolodner, D. Bensimon, and C. M. Surko, Phys. Rev. Lett. 60, 1723 (1988); J. J. Niemela, G. Ahlers, and D. S. Cannell, ibid. 64, 1365 (1990).

[50] H. Yahata, Prog. Theor. Phys. 85, 933 (1991); L. Z. Ning, Y. Harada, and H. Yahata, ibid. 96, 669 (1996).

[51] W. van Saarloos and P. C. Hohenberg, Physica D56, 303 (1992);

[52] S. Fauve and O. Thual, Phys. Rev. Lett. 64, 282 (1990);

[53] R. J. Deissler and H. R. Brand, Phys. Lett. A 146, 252 (1990);

[54] M. Bestehorn, R. Friedrich, and H. Haken, Phys. Rev. A42, 7195 (1990);

[55] H. Riecke, Phys. Rev. Lett. 68, 301 (1992); Physica D61, 253 (1992). 


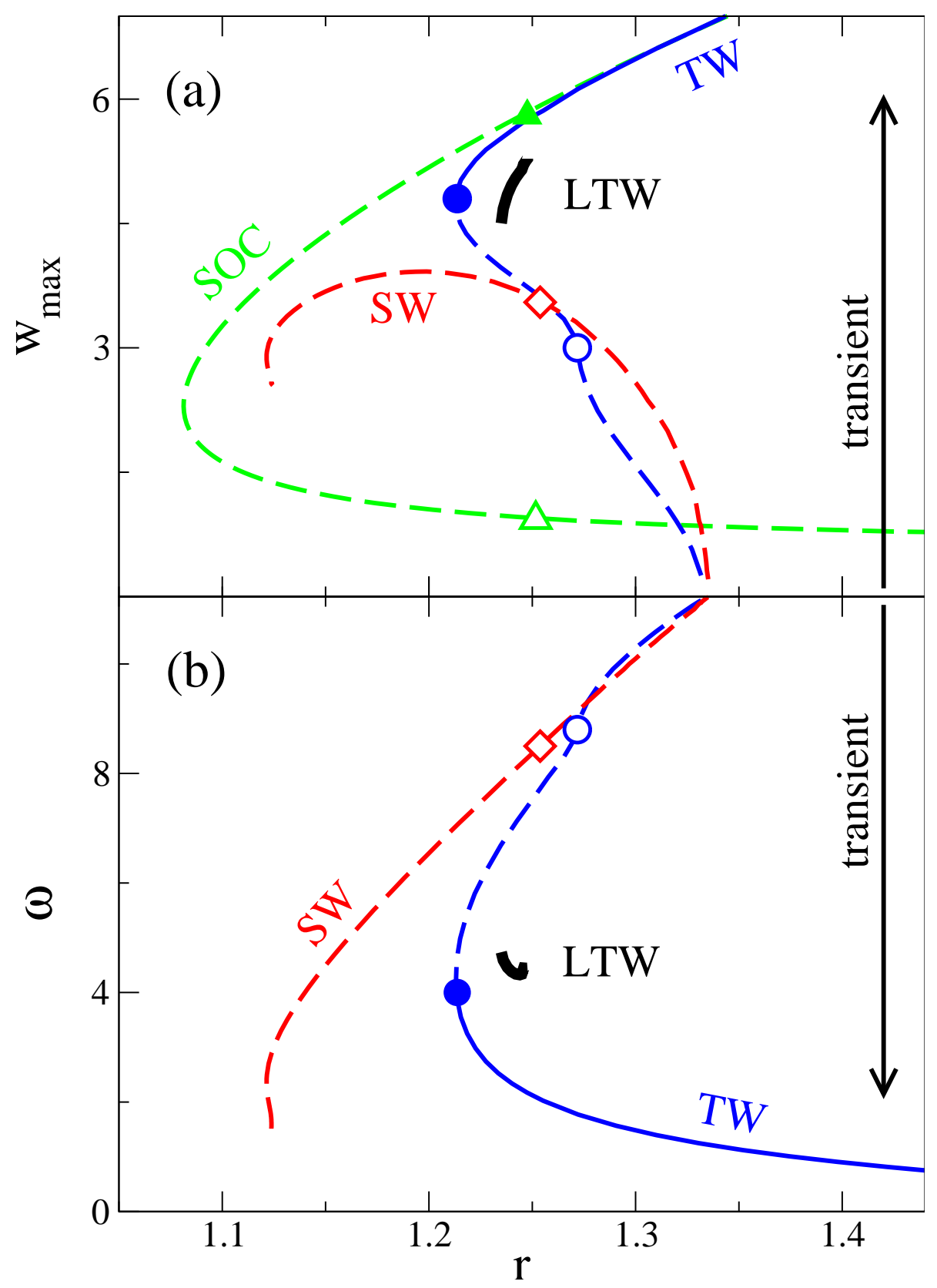

FIG. 1: Bifurcation properties of maximal vertical flow velocity $w_{\max }(\mathrm{a})$ and frequency $\omega(\mathrm{b})$ versus $r$ for representative relaxed nonlinear convective SOC, TW, SW, and LTW states. Full (dashed) lines refer to stable (unstable) states. Symbols identify the states that are discussed in more detail. TW and SW solutions bifurcate subcritically out of the conductive state at the oscillatory threshold $r_{o s c}$ with common Hopf frequency. Vertical arrows indicate the supercritical transient of Fig. 5 from a growing SW perturbation of the quiescent conductive state into a nonlinear TW. The wavelength of SOC, TW, and SW is $\lambda=2$. Parameters are $L=0.01, \sigma=10, \psi=-0.25$. 


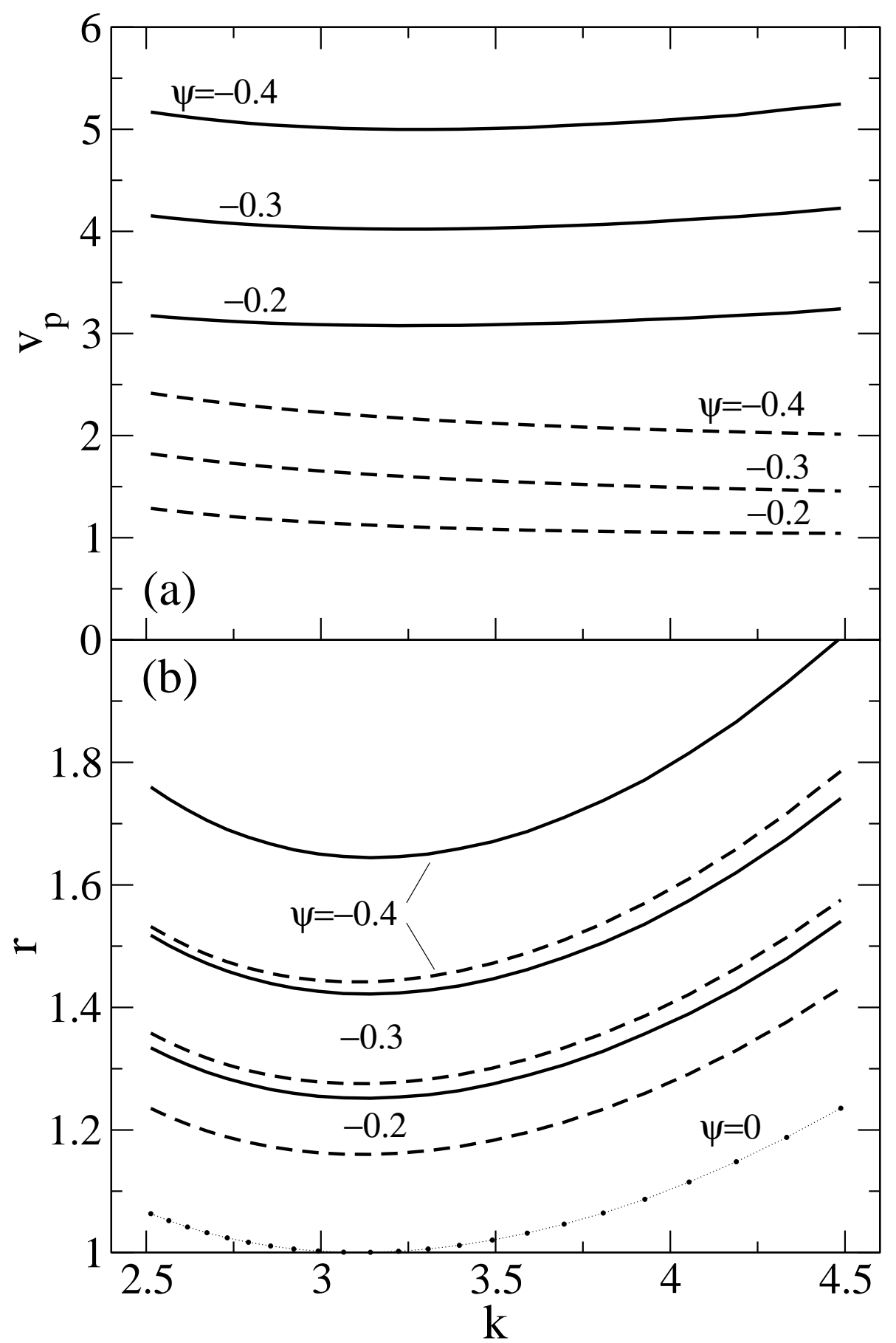

FIG. 2: Wave number dependence of TWs at onset and at their saddle-nodes for different $\psi$. (a) Phase velocities $v_{p}(k)$ at the onset (straight lines) and at the saddle (dashed lines). (b) Location of the onset $r_{\text {osc }}(k)$ (straight lines) and of the saddle-nodes $r_{s}^{T W}(k)$ (dashed lines) for TW branches in the $k-r$-plane. The dotted line marks the onset of SOCs in pure fluids. Parameters are $L=0.01$, $\sigma=10$. 


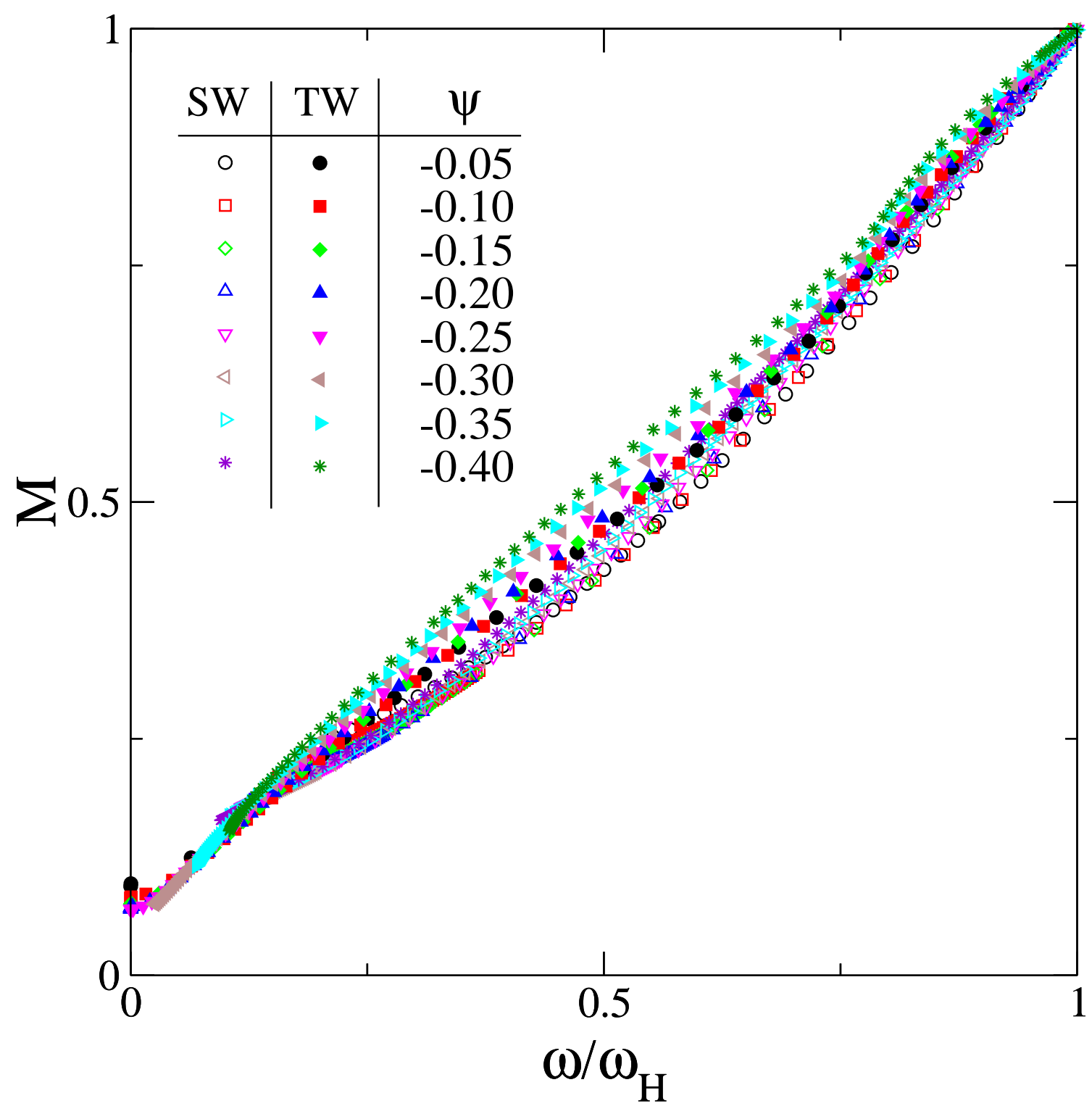

FIG. 3: Mixing number $M$ versus reduced oscillation frequencies of TWs and SWs for various Soret coupling $\psi$. For SWs the time averaged mixing number is plotted. Here $\omega_{H}$ is the respective Hopf frequency at onset. Parameters are $\lambda=2, L=0.01, \sigma=10$. 

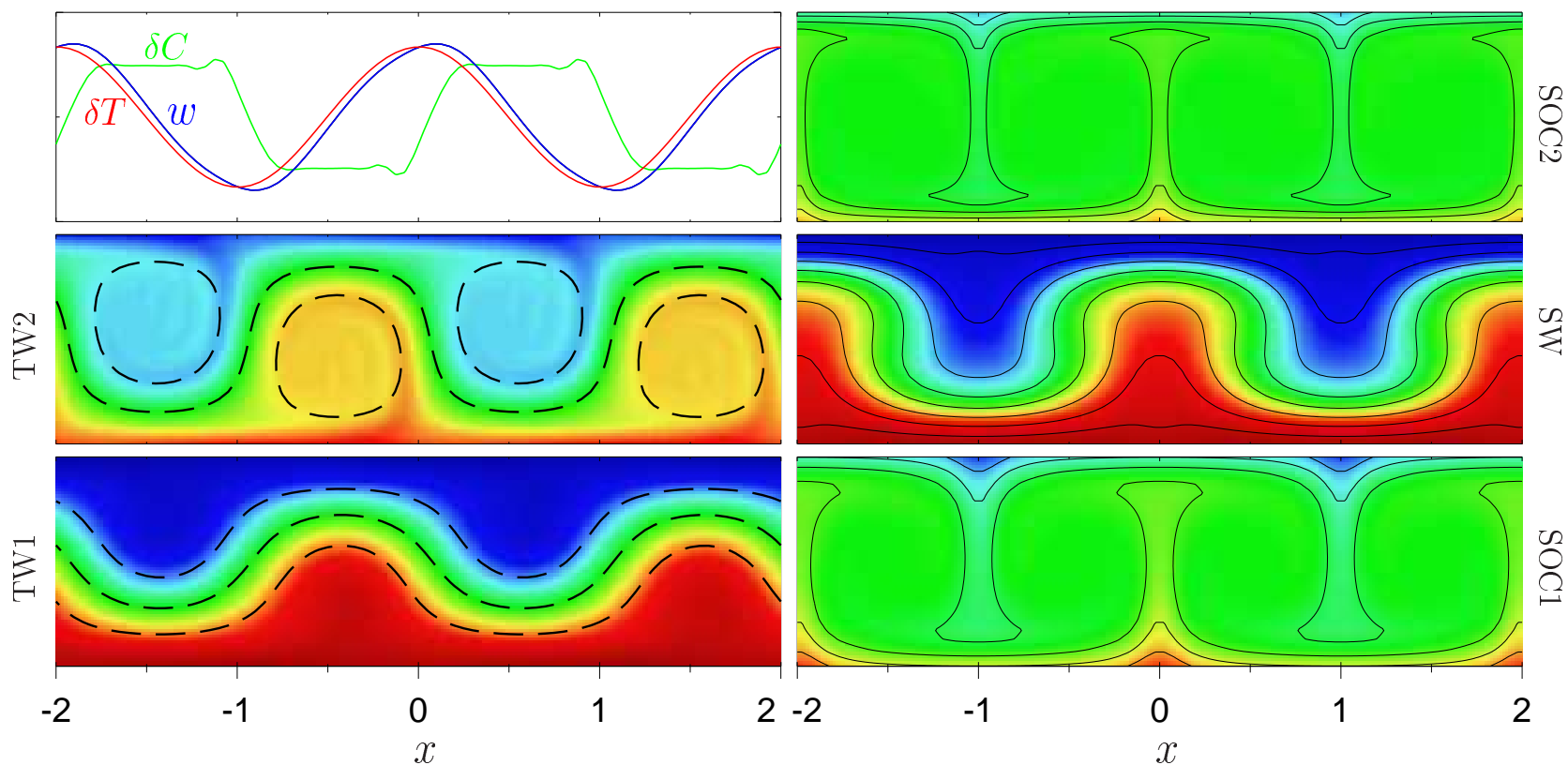

FIG. 4: Concentration distribution of selected extended states in vertical cross-sections through the rolls. TW1(2) and SOC1(2) are states marked by open (filled) circles and triangles, respectively, in Fig. 11 The SW refers to the lozenge in Fig. 1. The top figure in the left column shows the lateral wave profiles of $\delta C, \delta T$, and $w$ at midheight, $z=0$, of the fluid layer in the TW2. Parameters are $\psi=-0.25, L=0.01, \sigma=10, \lambda=2$. 

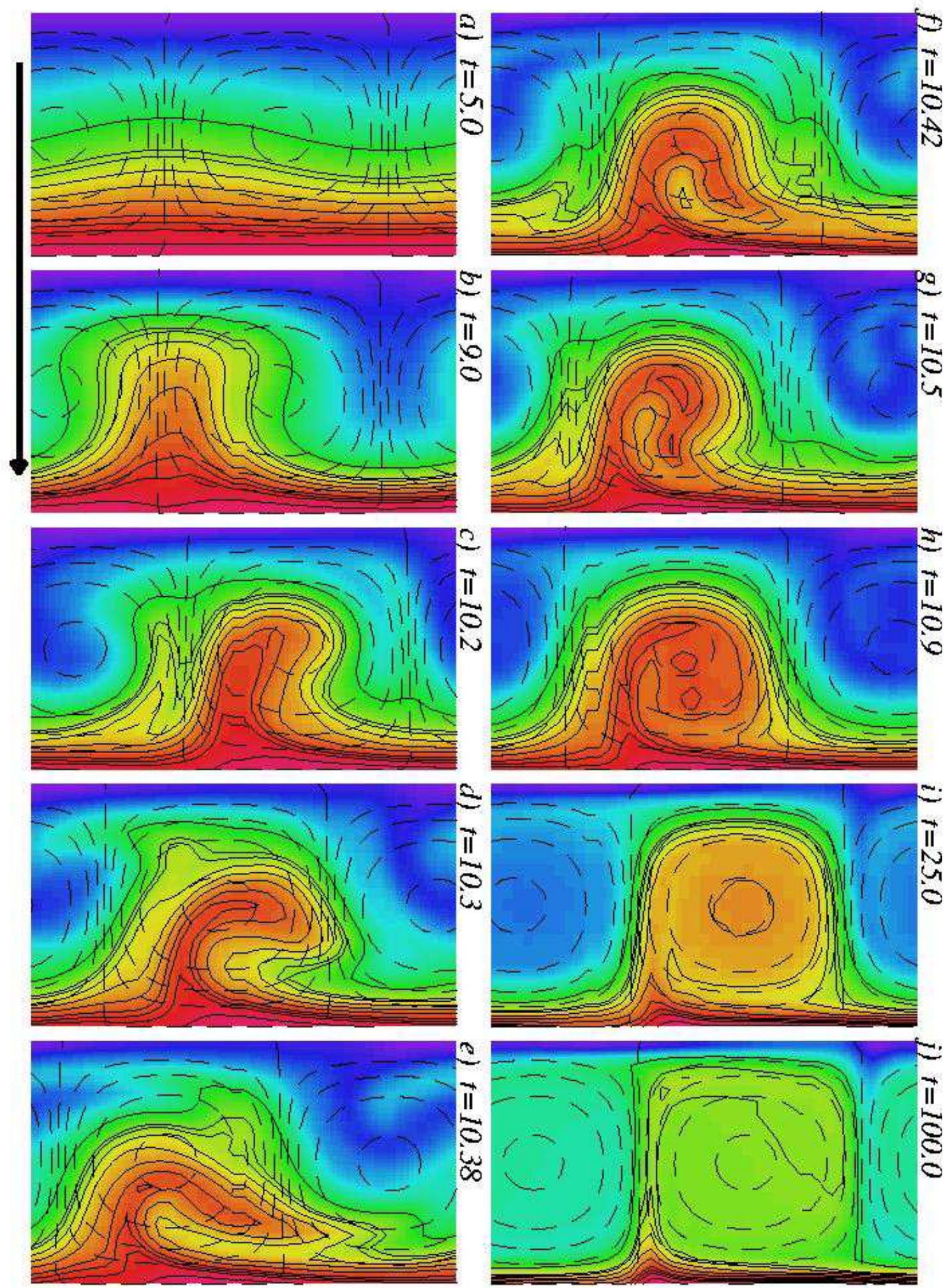

FIG. 5: Time ordered snapshots of the concentration redistribution in the $\mathrm{SW} \rightarrow \mathrm{TW}$ transformation. The concentration distribution in a vertical cross section of the fluid layer is displayed for one wavelength. Full isoconcentration lines are shown for $\delta C>0$. Dashed lines are streamlines, i.e., tangents to the instantaneous velocity field. The final TW $(\mathrm{j})$ propagates to the left. Parameters are $L=0.01, \sigma=10, \psi=-0.25, r=1.42$, and $\lambda=2$. 
Febru

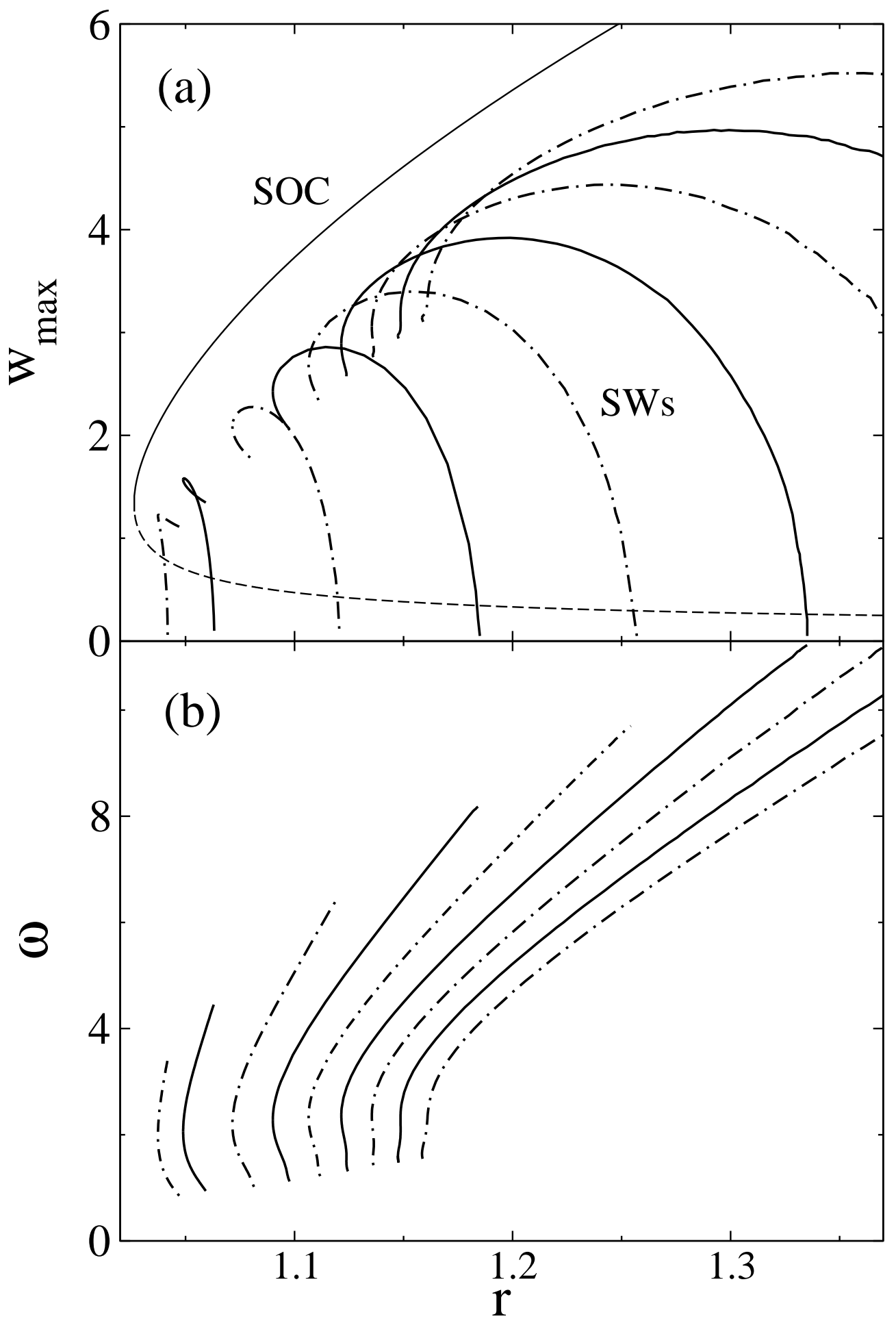

FIG. 6: Bifurcation properties of SWs for $\psi=-0.03,-0.05,-0.1,-0.15,-0.2,-0.25,-0.3,-0.35,-0.4$ (from left to right) : (a) Maximal vertical velocity $w_{\max }$. (b) Frequency $\omega$. Unstable SWs bifurcate subcritically out of the quiescent conductive state [lower ends of the curves in (a); upper ends in (b)] and undergo stability changes via saddle-node bifurcations. The SOC solution branch is shown for the sake of clarity only for $\psi=-0.03$. SOC curves for the other $\psi$ are shifted slightly to the right. Parameters are $L=0.01, \sigma=10$, and $\lambda=2$. 

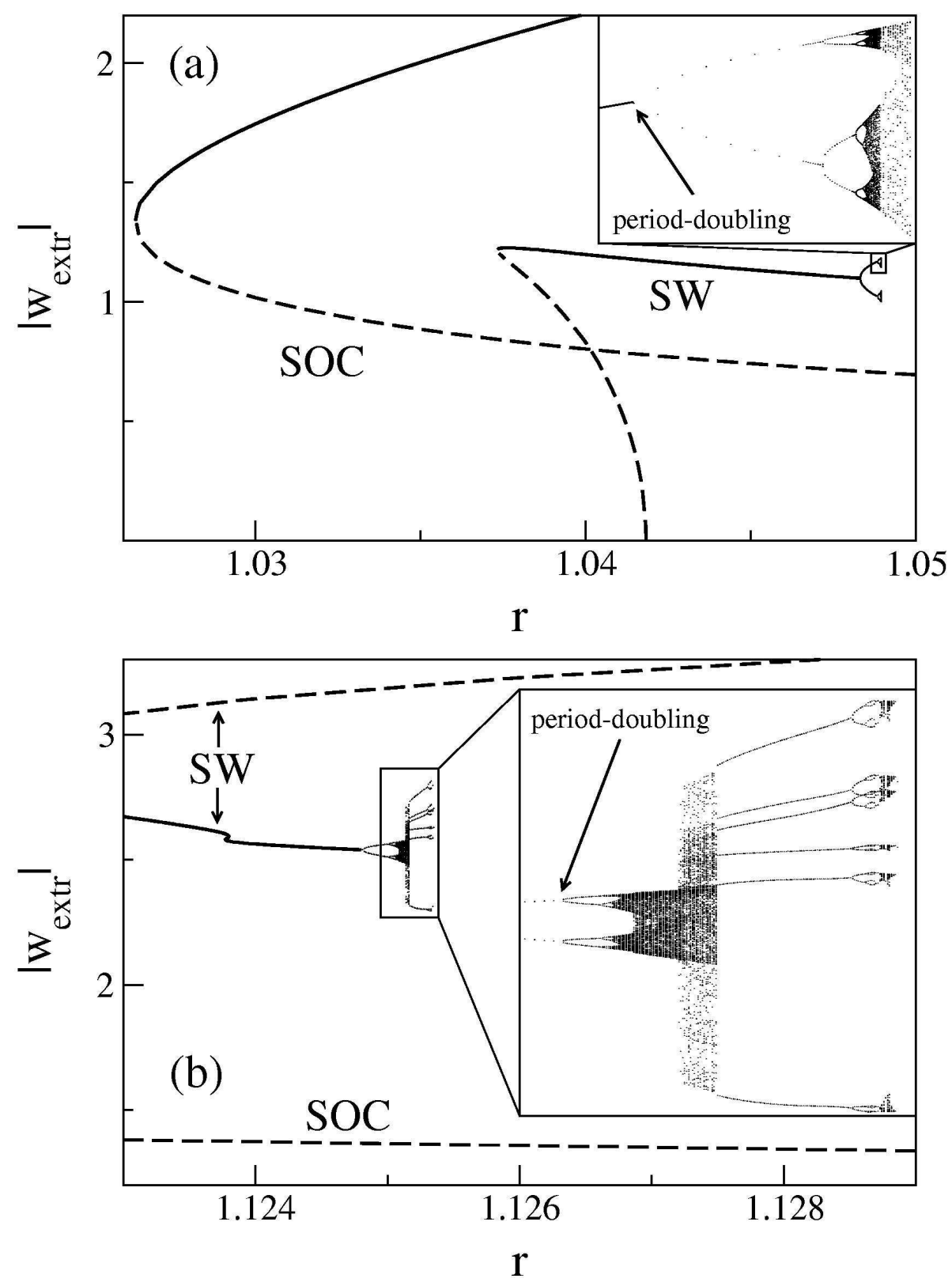

FIG. 7: Details of the SW bifurcation behavior for $\psi=-0.03$ (a) and $\psi=-0.25$ (b). The magnitude $\left|w_{\text {extr }}\right|$ of the extrema in the vertical flow is shown versus the reduced Rayleigh number $r$. The SWs bifurcate subcritically out of the quiescent conductive state at $r_{\text {osc }}$. When phasepinning conditions are applied they become stable (solid lines) at saddle-nodes positions [lying outside the plot range of (b)]. When the MTS is broken, the solid SW line splits into two since the magnitudes of the vertical flow extrema occurring during one oscillation cycle become different [see, e.g., Fig. 8 (b) where the downflow at $x=0=z$ is more intense than the upflow]. This MTS-broken SW starts at the arrows to undergo a period-doubling scenario (cf. insets) leading to chaos. Parameters are $L=0.01, \sigma=10$, and $\lambda=2$. 


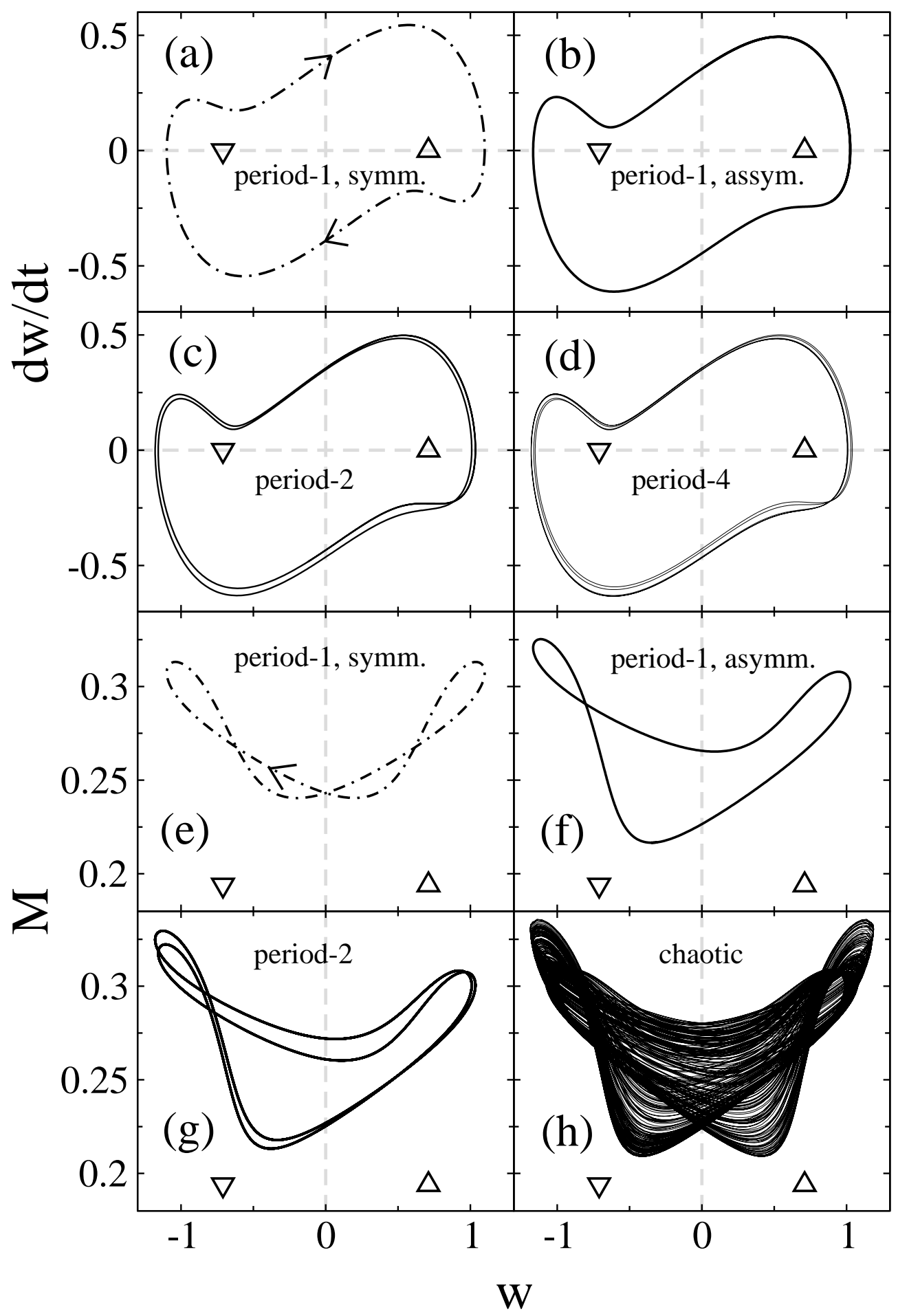

FIG. 8: MTS-breaking and period doubling in the phase space dynamics of SWs. Shown are $\dot{w}$ and the mixing number $M$ versus the vertical velocity $w$ at $x=0=z$ for $\psi=-0.03$. In (a)-(g) the full (dash-dotted) lines refer to asymmetric (symmetric) SWs for which the MTS is (not yet) broken. Period doubling is displayed in (b)-(d) and in (f)-(g). A chaotic trajectory is shown in (h). Upwards and downwards pointing triangles indicate symmetry degenerate unstable SOC fixed points with upflow and downflow, respectively, at $x=0$ that lie on the low-amplitude SOC solution branch [dashed line in Fig. Z(a)]. The flow velocity on the stable large-amplitude SOC solution 


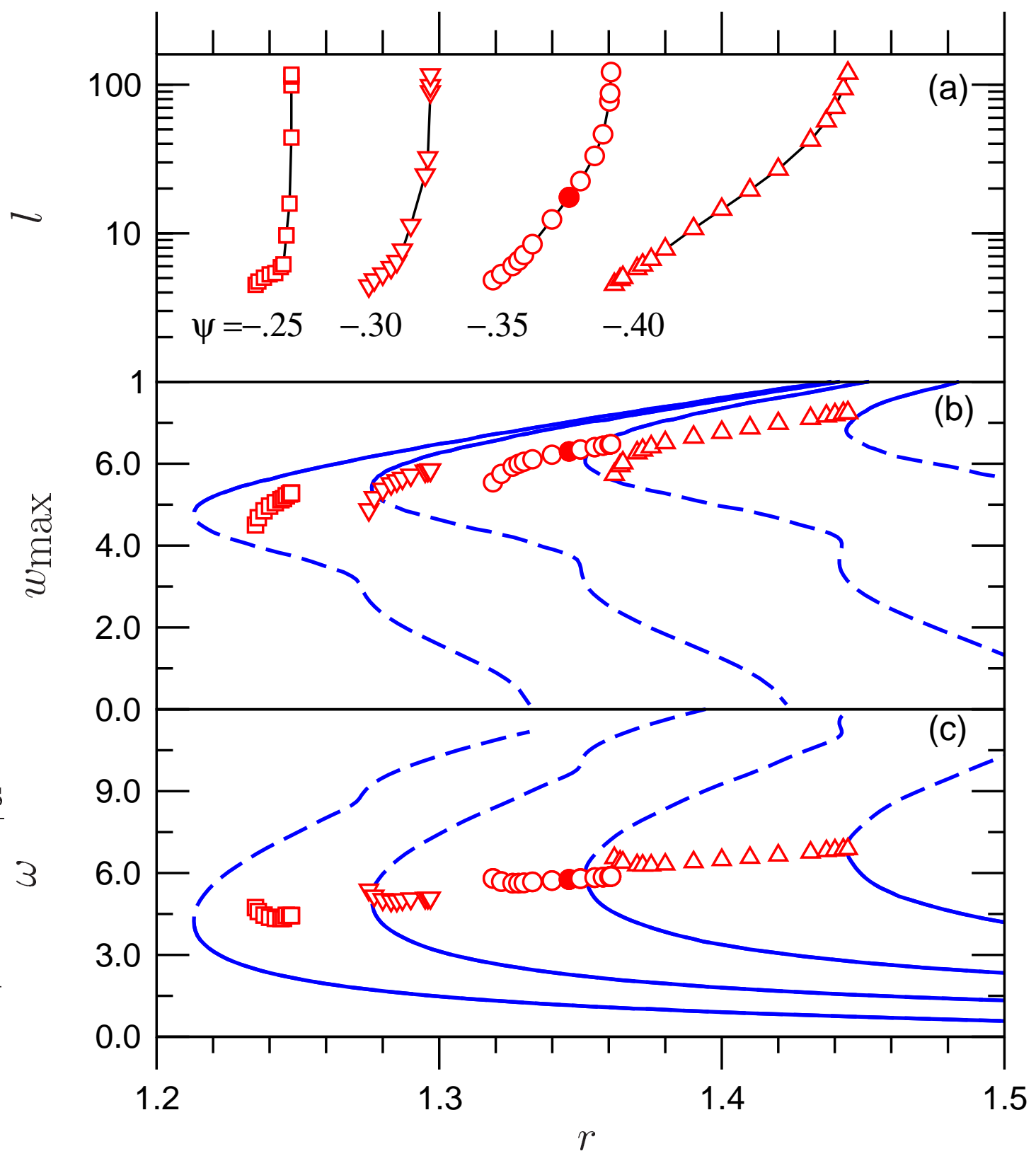

FIG. 9: Bifurcation properties of LTWs (symbols) and TWs (lines) for different separation ratios $\psi$ : (a) Full width $l$ of LTWs at half maximum of the envelope of the vertical velocity field $w$. (b) Maximal vertical flow velocity $w_{\max }$. (c) Frequency (for LTWs in the frame comoving with the drift velocity $v_{d}$ of the respective LTW). Filled circles identify the LTW whose structure is shown in Fig. 10, Lines in (b, c) denote TWs with saddle-node wave number $k_{s}^{T W} \simeq \pi$. Unstable TWs (dashed lines; determined with a control method) bifurcate subcritically with large Hopf frequency $\omega_{H}$ at $r_{\text {osc }}$ out of the conductive state and become stable (solid lines) at the saddle-node $r_{s}^{T W}$ when lateral periodicity is imposed with $\lambda=2 \pi / k$. Parameters are $L=0.01, \sigma=10$. 


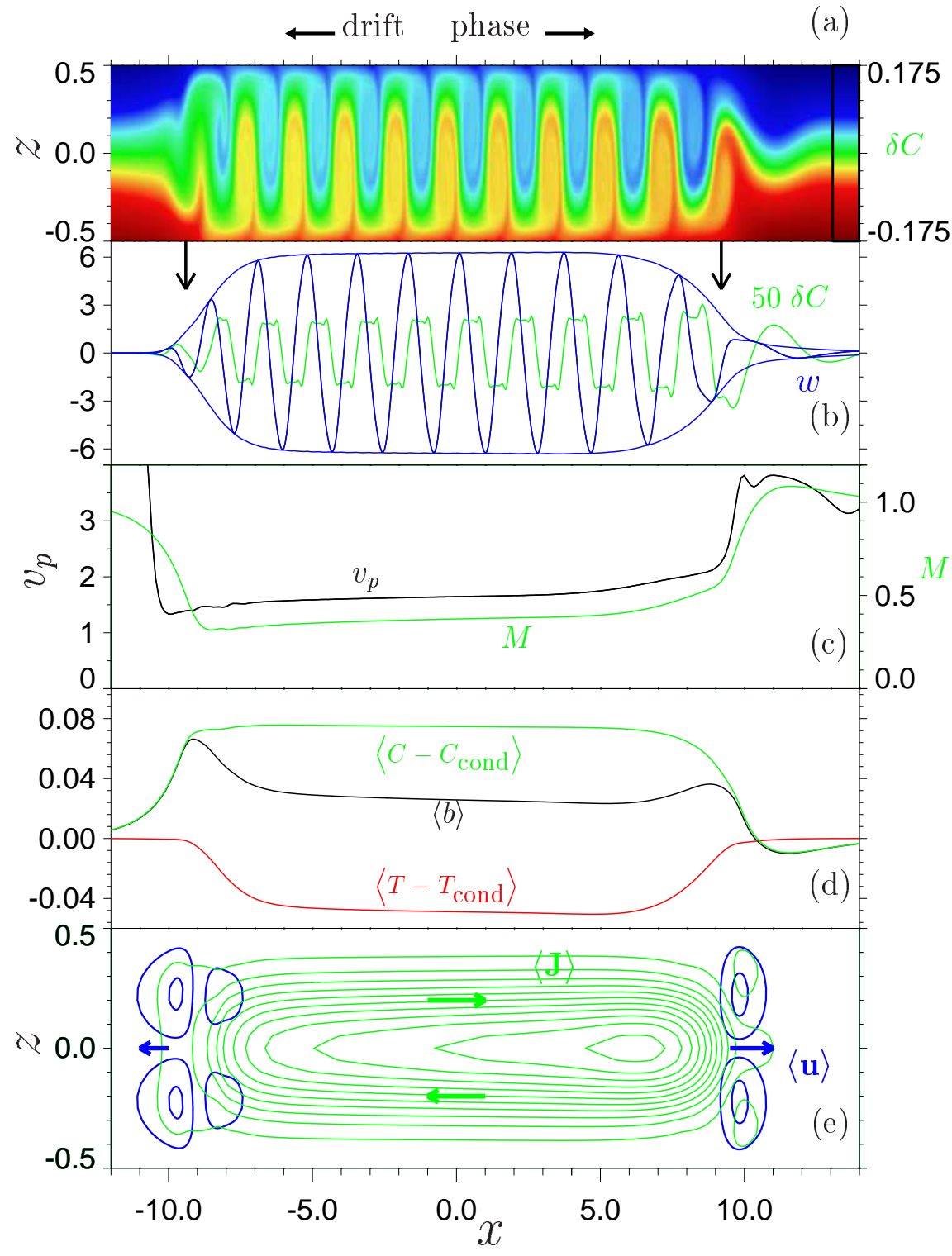

FIG. 10: Broad LTW of $l=17.4$ identified by filled circles in Fig. 9] (a) Snapshot of concentration deviation $\delta C$ from the global mean value in a vertical cross section of the layer. (b) Snapshots of lateral wave profiles at midheight, $z=0$, of $\delta C$, vertical velocity $w$, and its envelope. At the arrows $w_{\max }=v_{p}$. (b) Mixing number $M(x),\left[\mathrm{Eq}\right.$. (4.1)] and phase velocity $v_{p}$ of nodes of $w(z=0)$ in the frame comoving with the small drift velocity $v_{d}$ of the LTW. The variation of $\lambda(x)=2 \pi v_{p}(x) / \omega$ is the same since the LTW frequency $\omega$ is a global constant. (d) Time averaged deviations from the conductive state at $z=-0.25$ for concentration, temperature, and their sum, $b=T-T_{\text {cond }}+C-C_{\text {cond }}$, measuring the convective contribution to the buoyancy. (e) Streamlines of time averaged velocity field $\langle\mathbf{u}\rangle$ and concentration current $\langle\mathbf{J}\rangle=\langle\mathbf{u} \delta C-L \nabla(\delta C-\psi \delta T)\rangle$. $\langle\mathbf{u}\rangle$ results from $\langle b\rangle$ and affects $\langle\mathbf{J}\rangle$ via the contribution $\langle\mathbf{u}\rangle\langle\delta C\rangle$. In the upper half of the layer positive $\delta C$ (alcohol surplus) is transported to the right. In the lower half of the layer negative $\delta C$ (water surplus) is also transported to the right - positive $\delta C$ is transported there to the left as indicated by the arrow. 